\title{
Síndrome de Takotsubo: a propósito de un caso
}

\author{
Molero Silvero E. ${ }^{1}$, Borrego Jiménez P. ${ }^{2}$, Bartolomé Cela E. ${ }^{3}$, Rada Martínez I. ${ }^{4}$, Carretero Quevedo A. ${ }^{5}$
}

Sanid. mil. 2012; 68 (3): 168-171; ISSN: 1887-8571

\section{RESUMEN}

Se presenta el caso de una mujer de 72 años de edad, ingresada en la UCI con diagnóstico de Síndrome Coronario Agudo Sin Elevación del ST (SCASEST). El cateterismo cardíaco demostró la integridad de su árbol coronario, siendo diagnosticada finalmente de Síndrome de Takotsubo.

PALABRAS CLAVE: Takotsubo, Cardiomiopatía por estrés, Síndrome coronario agudo.

\section{Tako-tsubo syndrome: report of a case}

SUMMARY

This report presents the case of a 72 years-old woman, who was admitted in ICU with a diagnostic of non-ST-segment elevation acute coronary syndrome (NST-ACS). Cardiac catheterization showed a normal coronary angiogram, and the final diagnostic was TakoTsubo Syndrome.

KEY WORDS: Tako-tsubo, stress myocardial injury, Acute coronary syndrome.

\section{INTRODUCCIÓN}

Se estima que el Infarto Agudo de Miocardio (IAM) con coronarias normales representa, según las series, aproximadamente el 2-3\% de todos los IAM que son sometidos a coronariografía ${ }^{1}$. Ha sido ampliamente descrito en la literatura pero aún así, no siempre es reconocido a tiempo, lo que supone la realización de procedimientos innecesarios y, a veces, la administración de un tratamiento no apropiado para dicha entidad. Con este caso clínico, repasamos las características clínicas, diagnósticas y terapéuticas del Síndrome de Takotsubo (STT), cuya incidencia se estima en un $1-2 \%$ de todos los síndromes coronarios agudos $(\mathrm{SCA})^{2}$.

\section{OBSERVACIÓN CLÍNICA}

Mujer de 72 años, con intolerancia digestiva a los corticoides, sin alergias farmacológicas conocidas, con hipertensión arterial en tratamiento con Irbesartán y con intenso estrés. Acude al Servicio de Urgencias por un cuadro de dolor torácico típico de isquemia miocárdica, de localización precordial, opresivo, que no se modifica con los movimientos posturales ni respiratorios, irradiado hacia región dorsal y extremidades superiores, con sensación parestésica en los mismos, de 8-9 horas de evolución. Esa misma mañana había presentado un

\footnotetext{
${ }^{1}$ Lda. Medicina. Servicio de Medicina Intensiva.

2 Cap. Médico. Servicio de Medicina Intensiva.

3 Tcol Médico. Servicio de Medicina Intensiva.

${ }^{4}$ Tcol. Médico. Servicio de Cardiología.

5 Col. Médico. Servicio de Medicina Intensiva.

Hospital Central de la Defensa Gómez Ulla. Madrid. España.
}

Dirección para correspondencia: Esperanza Molero Silvero. Servicio de Medicina Intensiva. Hospital Central de la Defensa Gómez Ulla. Glorieta del Ejército, 1. 28047 Madrid. España. emolsil@oc.mde.es

Recibido: 12 de diciembre de 2011

Aceptado: 25 de abril de 2012 cuadro diarreico autolimitado con medidas conservadoras en domicilio. La paciente refiere 2 cuadros similares de dolor en último mes, con resolución espontánea.

La exploración física no aporta datos relevantes.

Durante su ingreso en Urgencias, se le realiza hemograma y bioquímica con resultado de leucocitosis (13.540) y un incremento de Troponina T 0,43 ng/dl. Un electrocardiograma (ECG) no evidenció hallazgos significativos (Fig. 1) y la radiografía de tórax no mostraba imágenes patológicas.

El ecocardiograma, objetivó aquinesia de los segmentos medio y apical de la cara septal, así como de los segmentos medio y apical de cara lateral, con un ventrículo izquierdo con aspecto de remodelado y un patrón compatible con disfunción diastólica.

Con estos datos se llega al diagnóstico probable de SCASEST, instaurando tratamiento con nitroglicerina, doble antiagregación, anticoagulación, antihipertensivo, beta-bloqueante y estatina e ingresada en la Unidad de Cuidados Intensivos (UCI).

El ECG al ingreso en la UCI presenta aplanamiento de onda T en V3-V4-V5. La coronariografía dio como resultado arterias coronarias epicárdicas sin estenosis angiográficas significativas, disquinesia de segmentos mediales en caras anterior, lateral, septal y diafragmático, con una reducción de la Fracción de Eyección de Ventrículo Izquierdo (FEVI) 30-35\%, con diagnóstico probable de Síndrome de Takotsubo (Fig. 2).

La evolución fue favorable y el ecocardiograma de control 8 días después del primero, mostró una hipertrofia ventricular concéntrica ligera con alteración de la relajación, siendo el resto de la exploración normal, por lo que se procede al alta hospitalaria con tratamiento ansiolítico.

\section{DISCUSIÓN}

El Síndrome de Takotsubo es una entidad descrita en Japón en 1990 por Sato $^{3}$. No fue hasta 2001, con un estudio de Tsuchihashi 


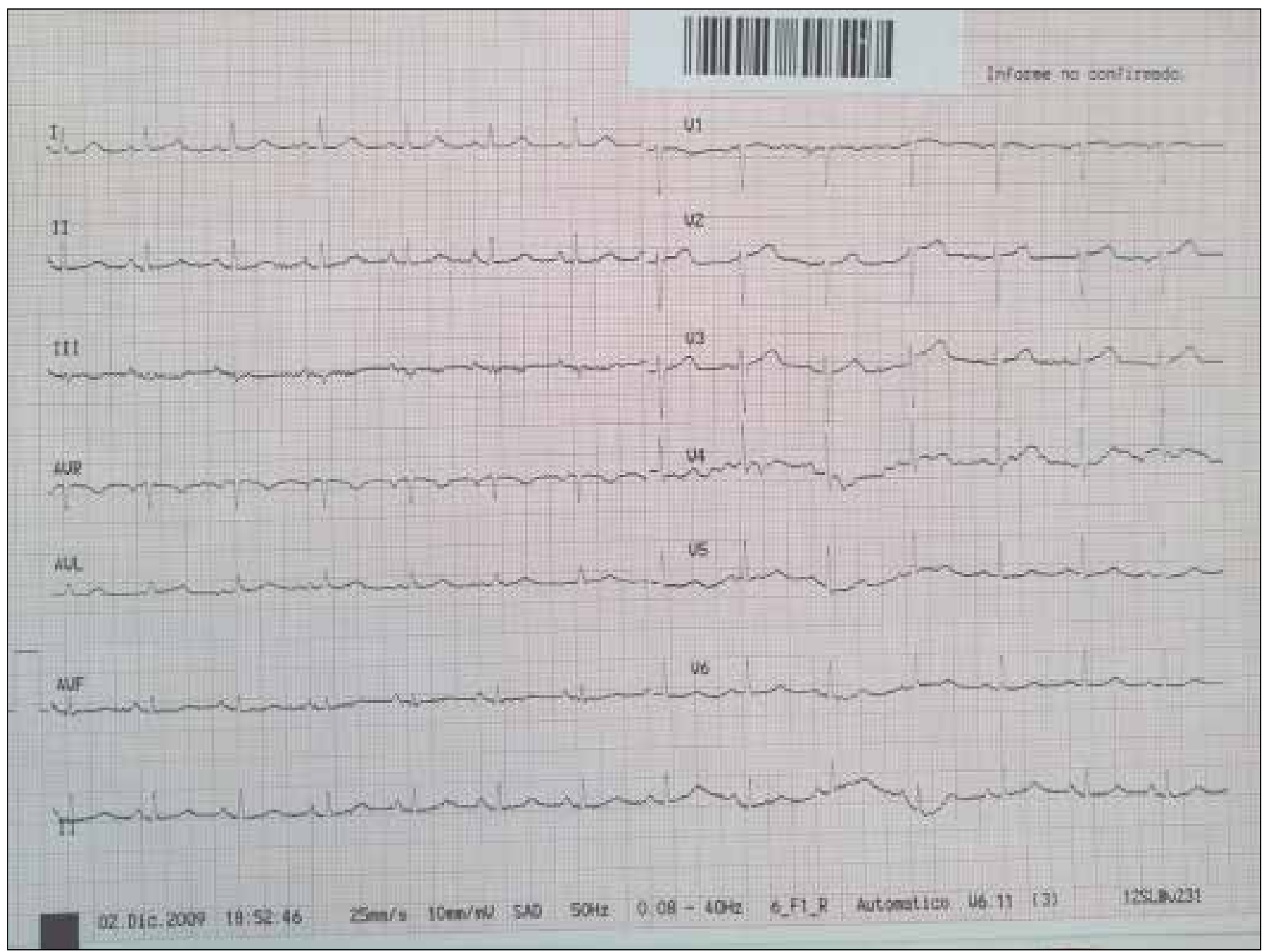

Figura 1. Electrocardiograma realizado a la llegada de la paciente al Servicio de Urgencias.

con 88 pacientes ${ }^{4}$, cuando se definió dicha patología. Su nombre se debe a la forma que adquiere el ventrículo izquierdo, que recuerda a las vasijas empleadas en Japón para la pesca de pulpos. Es una entidad que afecta principalmente a mujeres ${ }^{2,4-7}$ con edades superiores a los 55 años que se ven sometidas a un alto grado de ansiedad, bien sea físico (por ejemplo, durante la realización de una ecocardiografía de esfuerzo, descrita por López Cuenca et $\mathrm{al}^{8}$ ) o emocional ${ }^{6,9}, \mathrm{y}$ que puede estar relacionado en aproximadamente un $50 \%$ con hipertensión arterial ${ }^{2,10}$. Como posibles desencadenantes se han relacionado los antecedentes quirúrgicos, neoplasias endocrinas múltiples, Síndrome De Guillain Barré, sepsis, tirotoxicosis, obstrucción brusca de la vía aérea, etc.

Se han propuesto distintas teorías para explicar, sin éxito, la etiopatogenia del síndrome de Takotsubo (STT). La elevación brusca de catecolaminas, el espasmo y/o tortuosidad de las arterias coronarias, la tortuosidad de las arterias coronarias y alteraciones anatómicas de la arteria descendente anterior son algunas de las teorías estudiadas.

No se demostrado que la descarga catecolaminérgica secundaria a procesos como por ejemplo la hemorragia subaracnoidea, haya desencadenado un STT en el común de los pacientes ${ }^{11}$. La mayoría de los casos presentaban cifras de catecolaminas en rango normal o discretamente elevadas. La provocación de vasoespasmo corona-

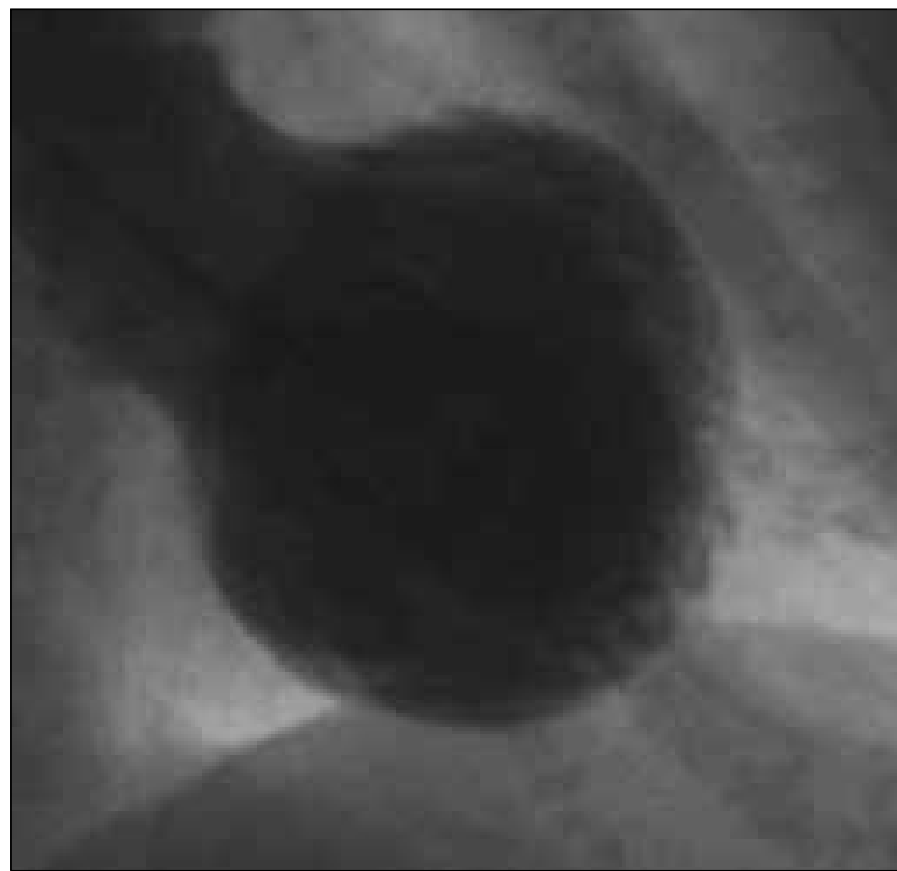

Figura 2. Ventriculografia con imagen de Takotsubo. 
rio $^{3,12}$ con Acetilcolina y Ergonovina ${ }^{4,5}$, se ha observado de forma variable en distintas series de pacientes. Una nueva hipótesis es la considerada por Gadda et al. ${ }^{13}$ que hace referencia a la existencia de descarga adrenérgica junto con una tortuosidad en diástole de las coronarias responsables y su conversión en un acodamiento máximo (kinking) en sístole que llega a «estrangular» la arteria comprometiendo el flujo. El aumento del consumo de oxígeno junto con el compromiso del flujo daría lugar a una zona de aturdimiento miocárdico (stunning) responsable de la disfunción focal ventricular. Por último, mencionar la posibilidad de la existencia de una arteria coronaria descendente anterior (DA) con gran segmento recurrente, lo cual tampoco se ha podido demostrar en el común de los pacientes. Ibañez et al. ${ }^{14}$ estudiaron la anatomía coronaria en un grupo de 11 pacientes diagnosticados de STT. Encontraron que en su muestra, los pacientes presentaban una DA de mayor longitud $(p=0,004)$ y con un segmento recurrente diafragmático más largo que el de los controles con coronarias normales $(\mathrm{p}<0,001)$.

Clínicamente, el 70-90\% presenta dolor torácico de características anginosas que puede ser irradiado e ir acompañado de cortejo vegetativo. Un porcentaje mucho menor debuta con disnea súbita y de manera casi anecdótica con shock cardiogénico ${ }^{9,11}$.

Para llegar al diagnóstico, una de las pruebas a realizar es un electrocardiograma $(E C G)^{4,6,9}$. Schneider et al. ${ }^{15}$ estimaron que la elevación del segmento ST se presentaba en aproximadamente el $50 \%$ de los casos. Puede aparecer también una onda T negativa en precordiales y una onda Q. La radiografía de tórax puede ser normal o mostrar signos de edema agudo de pulmón (EAP) o insuficiencia cardiaca. Se objetiva una elevación de la Troponina T (TnT) en casi el $100 \%$ de los pacientes, y elevación de la creatínfosfokinasa en el 50-60\%. Dicha elevación es menos marcada que en el SCA y la normalización ocurre en pocos días, a diferencia del descenso más lento que se observa en el IAM. La ecocardiografía $a^{4,5,16}$ realizada en la fase aguda suele mostrar un patrón característico y llamativo de hiperquinesia basal acompañada de una disquinesia o aquinesia de los segmentos medio y/o apical del ventrículo izquierdo. Si por el contrario observamos un patrón de aquinesia basal denominamos a esta variante Síndrome de Takotsubo inverso, descrito en el trabajo de Carrero Lérida et al. ${ }^{11}$. La coronariografía ${ }^{3-4,9,12}$ mostrará un flujo coronario arterial sin interrupciones, siendo el conjunto de los resultados de la clínica, la coronariografía, las alteraciones segmentarias de la contractilidad y la benignidad del cuadro lo que nos apoya el diagnóstico de STT y permite realizar un diagnóstico diferencial con otros tipos de IAM con coronarias normales. La ventriculografía $^{3,12,17-18}$ muestra un ventrículo izquierdo con la forma característica de vasija.

Según los expuesto anteriormente, la Clínica Mayo propuso una serie de criterios para el diagnóstico de STT:

1. Discinesia, acinesia o hipocinesia del VI; en ocasiones existe una situación estresante desencadenante, aunque no siempre;

2. Ausencia de enfermedad coronaria obstructiva o evidencia angiográfica de rotura de placa aguda;

3. Alteraciones nuevas en el ECG (elevación del ST y/o inversión de onda T) o modificación modesta de la troponina;

4. Ausencia de Feocromocitoma y/o Miocarditis ${ }^{19}$.

Algunos autores han realizado estudio de Medicina Nuclear con Tecnecio $99^{10}$, demostrando una zona afectada con abalonamiento apical que tras 3 meses desaparece por completo. Laraudogoitia et al. ${ }^{20}$ observaron mediante CardioResonancia (CardioRMN) una ausencia de realce tardío de Gadolinio, responsabilizándose de este hecho a la inflamación y edema presentes. Ingo Eitel et al. ${ }^{21}$ realizaron estudios de RMN en 256 pacientes con STT. Observaron una gran diversidad de patrones de contracción durante la fase aguda, siendo la más común la discinesia apical con acinesia de los segmentos apical y medioventricular del VI. El 17\% de los pacientes presentó una variante medioventricular con afectación apical y el 1\% una dilatación basal aislada. Un 34\% asoció además alteración del ventrículo derecho (VD) estando relacionado con una mayor estancia hospitalaria, menor FEVI y edad avanzada. En conclusión, los pacientes con afectación biventricular pueden presentar un curso más largo y severo de la enfermedad en comparación con los pacientes que presentan sólo afectación de VI. Las biopsias miocárdicas para el diagnóstico diferencial del STT con una miocarditis ${ }^{5}$, demuestran la (presencia de) pérdida focal de miocitos, infiltrado mononuclear leve y bandas de contracción sin necrosis ${ }^{10}$, al contrario de lo que esperaríamos encontrar en una miocarditis: infiltrado de leucocitos, fibrosis en grado variable y presencia, casi obligatoria de necrosis y/o degeneración. Además, Kurisu et al. $^{5}$, no hallaron títulos elevados de ningún agente infeccioso implicado. El grupo de Carrero ${ }^{11}$ realizó una prueba de Gated-SPECT a propósito de un caso de STT y determinaron que su práctica resultaba útil en la determinación de la Fracción de Eyección del VI, la normalidad o no de la perfusión miocárdica y los patrones de movilidad de las diferentes partes del VI.

Como posibles diagnósticos diferenciales ${ }^{9,11,16,22}$, debiéramos plantearnos, el SCA, miocardio aturdido ( dido demostrar mediante angiografía coronaria la obstrucción miocárdica durante la elevación del ST en el ECG), Angina Variante o de Prinzmetal (ya comentada la no provocación de espasmo de todos los pacientes con Acetilcolina/Ergonovina), estado catecolaminérgico, consumo de cocaína y la ya comentada miocarditis.

En cuanto al tratamiento ${ }^{5-6,9}$, no se obtienen datos que hayan sido extraídos de ningún ensayo clínico, pero la experiencia aconseja evitar la fibrinolisis que se aplicaría a los pacientes con SCA en centros sanitarios sin posibilidad de Hemodinámica. Debemos tener en cuenta, que ante una elevación del segmento ST con dolor típico anginoso y modificación de enzimas cardiacas, el tratamiento de elección es el trombolítico si no contamos con servicio de Hemodinámica, puesto que la gran mayoría de los pacientes con estas características, tendrán una lesión coronaria crítica. Se aconseja la antiagregación plaquetaria y/o administración intravenosa de heparina en la fase aguda ${ }^{23}$. La administración de Beta-Bloqueantes junto con Inhibidores de la Enzima Convertidora de Angiotensina en los pacientes hipertensos ha demostrado beneficios en estos pacientes. Debe mantenerse aproximadamente entre 3 y 6 meses, aunque la duración del tratamiento es motivo de controversia ${ }^{23}$. En algunos casos, la clínica y la hemodinámica del paciente obliga a la instauración de tratamiento con aporte de fluidoterapia, fármacos vasoactivos e inotrópicos como Levosimendán o Fenilefrina ${ }^{23}$. La hipervolemia puede desencadenar o exacerbar una IC preexistente. Cabe mencionar el tratamiento ansiolítico, necesario para mitigar el estrés desencadenante de esta dolencia. Pfister et al. ${ }^{23}$ aconsejan la administración de anticoagulación como prevención tromboembólica en todos aquellos pacientes con disfunción persistente de VI. 


\section{Síndrome de Takotsubo: a propósito de un caso}

La aparición de shock cardiogénico apoya en estos casos el uso de balón de contrapulsación intraaórtico.

Las complicaciones más frecuentes son el EAP y la IC en grado variable, seguidas por el shock cardiogénico, la bradicardia severa y las arritmias ventriculares y, de manera anecdótica, el accidente cerebro vascular de origen embólico 9,11,16. A los dos o tres días de la fase aguda comienza la recuperación clínica y hemodinámica. Las alteraciones del ECG y de la ecocardiografía pueden durar días o semanas y en algunos pacientes se ha observado la existencia de una disfunción ventricular izquierda residual. Pese a este buen pronóstico, el STT presenta una mortalidad estimada del 1-2\% ${ }^{16}$ y una recurrencia del $3 \%{ }^{24}$. Cabrera et al. realizaron un estudio a largo plazo, observando la presencia de un $31 \%$ de ángor entre los pacientes con SCASEST sin lesiones coronarias en un plazo medio de cuatro años ${ }^{25}$.

\section{BIBLIOGRAFÍA}

1. Da Costa A, Isaaz K, Faure E, Mourot A, Cerisier A, Lamaud M. Clinical characteristics, aetiological factors and long-term prognosis of myocardial infarction with an absolutely normal coronary angiogram. Eur Heart J 2001; 22: 1459-1465.

2. Del Nogal Sáez et al. Síndrome de tako-tsubo. Discinesia transitoria del ventrículo izquierdo. Presentación de nuestra casuística. Med Intensiva. 2011;35(5):307311.

3. Sato et al. Stunnes myocardium with speciphic (Takotsubo-type) left ventriculographic configuration due to multivessel spasm. Clinical aspect of myocardial injury. From ischemia to heart failure. Kodama K. Hori M. Eds Kagakuhyyouronsya Co. Tokio. 1990; 56:-64.

4. Tsuchihashi K, et al. Transient left ventricular apical ballooning without coronary artery stenosis: a novel heart syndrome mimicking acute myocardial infarction. J Am Coll Cardiol 2001;38:11-8.

5. Kurisu S. et al. Time course of electrocardiographic changes in patients with Takotsubo syndrome: comparison with acute myocardial infarction with minimal enzymatic release. Cir J. 2004;68:77-81.

6. Sánchez Llanos et al. Síndrome de Tako Tsubo. Rev Clín Med Fam 2010;3(2):127130.

7. García Vicente et al. Infarto agudo de miocardio con coronarias normales. Revista Electrónica de Medicina Intensiva. Caso clínico nº 20. Vol 8 nº 3, marzo 2008.

8. López-Cuenca A., et al. Una rara complicación de la ecocardiografía de esfuerzo. Rev Esp Cardiol. 2011;64(1):82-3.

9. Katscher, W. et al. Síndrome Tako-Tsubo simulando infarto agudo de miocardio. Emergencias 2006;18:309-311.

10. Pacheco-Bouthillier, A. D. et al. Síndrome de takotsubo. Informe de un caso y revisión de la literatura. Cir Cir 2010;78:157-161.

11. Carrero Lérida, M.J. et al. Síndrome de discinesia apical transitoria (Tako-
Tsubo) que simula un infarto agudo de miocardio. Rev Esp Med Nucl. 2010 doi:10.1016/j.remn.2010.03.009

12. Dote et al. Myocardial stunning due to simultaneous multivessel coronary spasm: a review of 5 cases. J Cardiol 1991; 21:203-14.

13. Gadda, C. E. Cardiopatía inducida por estrés (Tako-Tsubo). Nueva hipótesis fisiopatológica. Rev Argent Cardiol 2010;78:43-45.

14. Ibañez B et al. Tako-tsubo y arteria coronaria descendente anterior. Rev Esp Cardiol 2004;57(3):209-16.

15. Scheider et al. Clinical management y therapeutic strategies in patients with Tako-Tsubo cardiomyopathy: Results of the German tako-tsubo registry. Circulation. 2009; 120:S890.

16. Salaverría Garzón, I. et al. Síndrome de Tako-Tsubo (discinesia apical transitoria). Un síndrome que simula un infarto de miocardio. An Med Interna (Madrid) 2008;25:20-22.

17. Citro et al. Tako-tsubo cardiomyopathy and chronobiology. J Am Coll Cardiol, 2010;55:500-501.

18. Obón Azuara, M. R. et al. Cardiomiopatía de Takotsubo: disfunción transitoria apical de ventrículo izquierdo. Med Intensiva. 2007;31(3):146-52.

19. Abe Y, Kondo M, Matsuoka R, Araki M, Dohyama K, Tanio H. Assessment of clinical features in transient left ventricular apical ballooning. J Am Coll Cardiol. 2003;4:737-42.

20. Laraudogoitia, E. et al. Utilidad de la resonancia magnética cardiaca en el diagnóstico de los pacientes con síndrome coronario agudo y coronarias normales. Rev Esp Cardiol. 2009;62(9):976-83.

21. Ingo Eitel et al, Clinical characteristics and cardiovadcular magnetic resonance findings in stress cardiomyopathy. JAMA 2011; 306(3):277-286.

22. González Rodríguez et al. Disfunción ventricular transitoria o síndrome de TakoTsubo. A propósito de un caso. Emergencias 2006;18:247-249.

23. Pfister S, Wagar P, Casserly IP. Stress-Related Cardiomyopathy in a 31-Year-Old Woman. AANA Journal 2010;78(5):406-11.

24. Núñez Gil et al. Cardiopatía de estrés o síndrome de Tako-Tsubo: conceptos actuales. Rev. argent. cardiol. Jun 2009;77(3):218-223.

25. Cabrera $\mathrm{C}$ et al. Acute coronary syndromes without significant coronary lesions: clinical profile and long term outcome. Abstract World Congress of Cardiology May 2008. Circulation 2008; 118; 12, e 258, p 539. 